\section{Prolonged Asthma Exacerbation as an Initial Presentation in Hereditary Hemorrhagic Telangiectasia}

Jacob Umscheid, M.D., ${ }^{1,2}$, Joshua Albright, D.O.,2, John Chazhoor, MS-41, Rhythm Vasudeva, M.D., M.S., ${ }^{1,3}$

${ }^{1}$ University of Kansas School of Medicine-Wichita, Wichita, KS ${ }^{2}$ Department of Pediatrics

${ }^{3}$ Internal Medicine/Pediatrics Residency Program Received Aug. 4, 2021; Accepted for publication Nov. 21, 2021; Published online Dec. 2, 2021 https: /doiorg/10.17161/ijm.voll4.15752

\section{INTRODUCTION}

Hereditary hemorrhagic telangiectasia (HHT), also known as Rendu-Osler-Weber syndrome, is an autosomal dominant disease of multiple pathological arteriovenous malformations (AVM) throughout the body. ${ }^{1}$ The malformations of HHT are fragile in nature and directly connect arterial blood flow to the venous vasculature, bypassing normally present capillary beds. Disease presentation is dependent on the location of these malformations in the body. Although typically seen as telangiectasias or small AVMs on the skin and mucosa, large AVMs and complications secondary to AVMs can present with symptoms involving the brain, lungs, and liver. As mentioned, the fragility of these vascular malformations lends a hand to deleterious consequences such as hemorrhage, vascular shunting, and passage of venous emboli to the brain. $^{2}$

Initially thought to be a very rare disease, genetic testing has shown the prevalence of HHT to be greater than previously believed. ${ }^{1}$ Prevalence for HHT is estimated to be $1 / 5,000$ to $1 / 10,000$ with equal distribution between gender and race..$^{3-5}$ Initial symptoms, such as petechiae and epistaxis, may be mild in presentation, and the actual prevalence may be greater than what can be measured. Severe features of HHT, such as pulmonary AVMs, can be the presenting features of HHT and have been observed in 15 to $35 \%$ of cases. ${ }^{6}$

In this article, a case of a young female is presented with prolonged acute asthma exacerbation discovered to have large pulmonary AVMs who showed improvement after endovascular coiling and eventual diagnosis of HHT.

\section{CASE REPORT}

A 17-year-old female with a past medical history of mild intermittent asthma presented to the pediatric emergency department with a chief complaint of cough, congestion, and wheezing that was unresponsive to albuterol. Her wheezing had started one day prior. Her past medical history included multiple episodes of community acquired pneumonia not requiring hospital admission and recurrent epistaxis. Family history was not available as her parents were not available at the time of presentation.

Initial vital signs included temperature of $37.4^{\circ} \mathrm{C}$, pulse of $128 \mathrm{bpm}$, respiratory rate of 20 breaths per minute, blood pressure of 139/81 $\mathrm{mmHg}$, and oxygen saturation of $85 \%$. Physical examination was significant for respiratory distress with suprasternal retractions and inspiratory and expiratory wheezing. Chest x-ray showed faint patchy airspace opacities in the right midlung (Figure 1). Viral respiratory PCR indicated infection from rhinovirus and enterovirus. Hypoxia improved with $4 \mathrm{~L}$ via nasal canula and the patient was transferred to the pediatric

\section{KANSAS JOURNAL of MEDICINE}

intensive care unit for management of acute asthma exacerbation triggered by a viral infection. She was initiated on scheduled albuterol for bronchodilation and corticosteroids for anti-inflammation and showed rapid improvement. On day two of hospitalization, she was maintaining an oxygen saturation above $90 \%$ on $2 \mathrm{~L}$ and was transferred to the pediatric floor for continued hypoxia management.

On day three of hospitalization, the patient developed worsening oxygenation and ventilation and a repeat chest X-ray revealed a persistent right middle lobe opacity, prompting initiation of amoxicillin-clavulanate for presumed community acquired pneumonia. She also developed epistaxis that resolved spontaneously and was switched to a high flow face mask due to presumed nasal mucosa irritation from nasal canula. The patient showed minimal improvement and persistent hypoxia despite prolonged treatment course. On day 10 of hospitalization, the patient's mother revealed a personal history of HHT and concern for a pulmonary arteriovenous malformation was investigated with computerized tomography angiogram (CTA). CTA revealed multifocal filling branches extending to the periphery of the right middle lobe with at least three major feeding AVMs present, expanding from directly adjacent to the bifurcation of the right middle lobe and right lower lobe bronchial arteries extending to the periphery (Figure 2). Echocardiogram demonstrated agitated saline contrast in the left atrium, indicating a right to left shunt consistent with a pulmonary AVM. Interventional radiology was consulted for correction of the pulmonary AVMs with three separate locations requiring coil and plug placement (Figure 3). Her hypoxia resolved immediately after this intervention.

An investigation for other AVMs was initiated with a CTA of the head and liver ultrasound, both with negative results. No telangiectasias were observed on the skin of the patient or on the oral mucosa. The genetics service was consulted and a presumptive diagnosis of HHT was made. Genetic testing was offered to confirm the diagnosis but was denied by family.

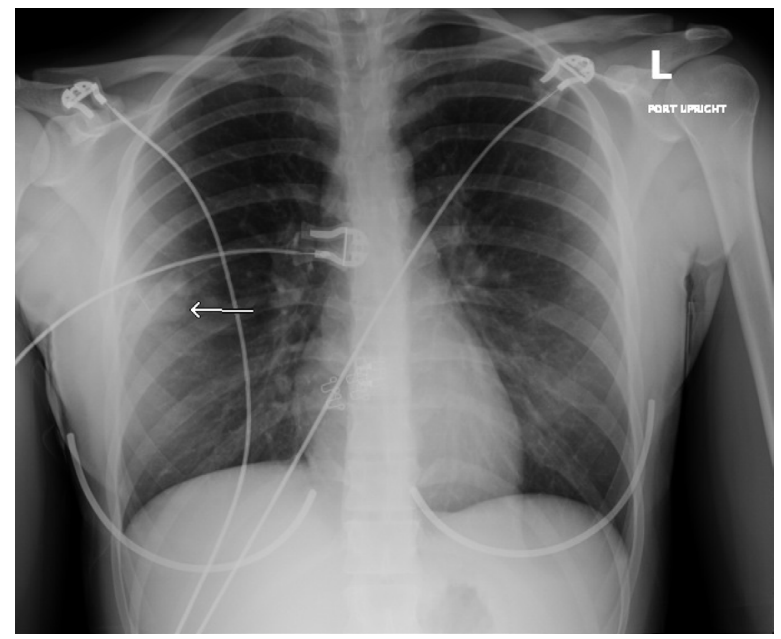

Figure 1. Admission AP chest X-ray showing right mid-lobe air space opacity. 
KANSAS JOURNAL of MEDICINE

HEREDITARY HEMORRHAGICTELANGIECTASIA continued.

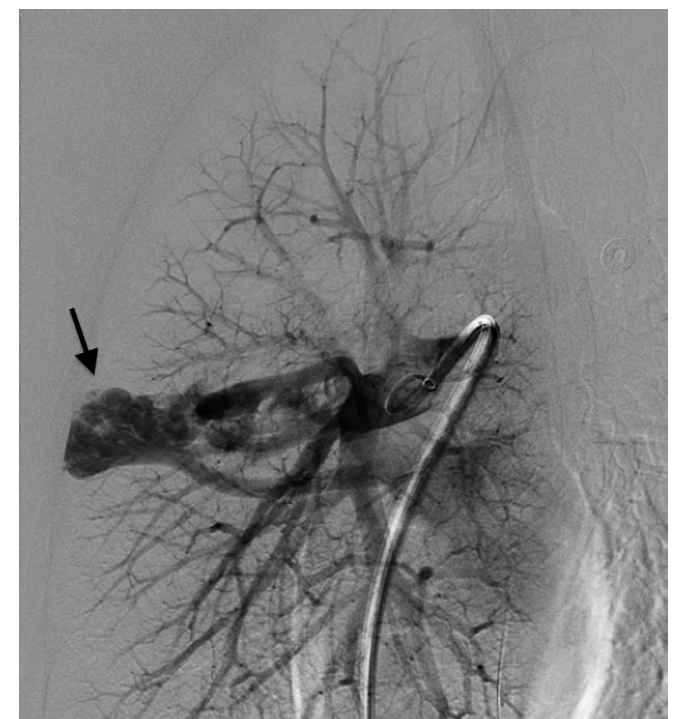

Figure 2. Pre-correction angiography imaging displaying large, complex pulmonary arteriovenous malformations of the right middle lobe.

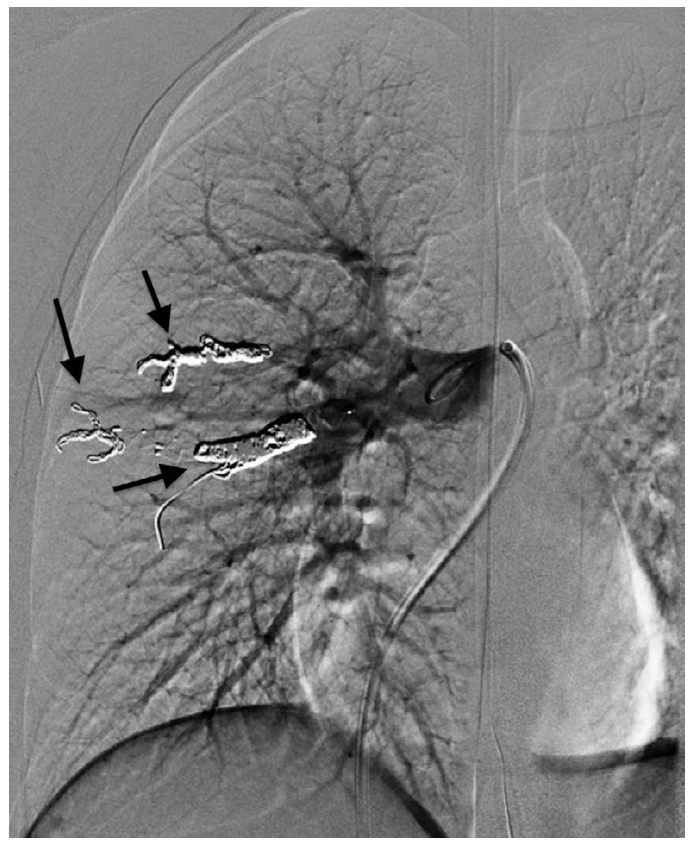

Figure 3. Post-correction angiography imaging showing coil and plug placement with satisfactory exlusion of the AVM with no appreciable residual filling.

\section{DISCUSSION}

This case demonstrated a presentation of a pulmonary AVM secondary to HHT resulting in prolonged recovery of an acute asthma exacerbation. Although epistaxis secondary to telangiectasias and involvement of the nasal mucosa is the most common presentation of HHT, these diagnoses can go unrecognized and dismissed as benign. ${ }^{5}$ When epistaxis is combined with a prolonged course of respiratory illness, investigation into HHT is warranted. Diagnosis of HHT in adolescents can be difficult, as severity and recurrence increase with age and initial episodes of epistaxis could be relatively mild and result in the underdiagnosis of HHT? The presentation of epistaxis often precedes other manifestations of HHT by 20 to 30 years, making it imperative to carry a low index of suspicion in patients presenting with recurrent epistaxis. ${ }^{8}$
In addition to telangiectasias, much larger AVMs in the pulmonary vasculature can contribute to significant morbidity of individuals with HHT. ${ }^{6}$ These consist of a direct connection between a branch of the pulmonary artery and a branch of the pulmonary vein with potential aneurysm at the point of convergence. Patients with HHT tend to present with multiple AVMs and are found most commonly bilaterally in the lower lobes of the lungs. ${ }^{9}$ Depending on the method of investigation, 60 to $90 \%$ of individuals with pulmonary AVMs have an underlying diagnosis of HHT., ${ }^{6,10}$ Detection of these underlying AVMs increases when utilizing tools such as high-resolution computed tomography (CT) and transthoracic contrast CT with saline contrast. ${ }^{1}$ Clinical manifestations, such as dyspnea, fatigue, or cyanosis, from pulmonary AVMs stem from the right-to-left shunt created and likely increase in severity depending on the number of AVMs.

Other concerning features of HHT include neurological and hepatic manifestations. Paradoxical emboli resulting in cerebral vascular accidents or abscesses can occur secondary to pulmonary shunting. ${ }^{11,12}$ Cerebral vascular malformations also can result in dural fistulas, cavernomas, and aneurysms. ${ }^{11}$ Because of this risk, guidelines recommended angio-magnetic resonance imaging screening to investigate cerebral vascular malformations in patients with definite HHT. ${ }^{5}$ The prevalence of cerebral AVMs in HHT was approximated to be $10 \%$ based on computed tomography, however, this is considered as an underestimate as there are more sensitive methods of investigation available. ${ }^{13}$ Akin to both pulmonary and central nervous system vasculature, hepatic vasculature in individuals with HHT also can be found to have AVMs. According to studies utilizing both CT and ultrasounds, frequency of hepatic vascular abnormalities were $74 \%$ when using CT and $41 \%$ when investigated using ultrasound. ${ }^{13,14}$ Only $8 \%$ of those studied were symptomatic prior to investigation. ${ }^{14}$ Complications of hepatic AVMs include the potential of inducing heart failure secondary to high cardiac output caused by left-to-right shunting within the hepatic vasculature, but also biliary disease and portosystemic encephalopathy. ${ }^{13,15}$

Until 2000, there were no standardized clinical diagnostic criteria for the diagnosis of HHT. A consensus statement on four criteria with an interpretation on the number of positive results and subsequent chances of positive diagnosis, named the Curaçao criteria, was made in 2000., ${ }^{516}$ These criteria are described in Table 1 . Three of the four criteria can be seen with history and physical exam alone. Since its initial release, studies have looked to verify the proposed Curaçao criteria. One study of 263 first-degree relatives who were carriers of disease-causing mutations used genetic testing as a gold standard and found that the Curaçao criteria had a sensitivity of $90.3 \%$ of the 186 with HHT causing mutations, $100 \%$ positive predictive value of firstdegree relatives, and negative predictive value of unlikely diagnosis with $97.7 \% .{ }^{12}$ In 2020, McDonald et al. ${ }^{17}$ reviewed the genetic testing results of 152 individuals who were diagnosed clinically using the Curaçao criteria and concluded approximate $97 \%$ presence of causative mutations of either ENG, ACVRL1, or SMAD4. 
Table 1. Curaçao criteria for the diagnosis of HH'T.

Epistaxis: Spontaneous and recurrent

and nose

Internal lesions: Such as GI telangiectasia, pulmonary AVM, hepatic AVM, cerebral AVM, and spinal AVM

Family history: A first-degree relative with HHT according to these criteria

The HHT diagnosis is definite if three or more criteria are present, possible or suspected if two are met, and unlikely if fewer than two criteria are present.

\section{CONCLUSIONS}

The diagnosis of HHT remains a clinical diagnosis through thorough medical history, family history, and physical examination. When the diagnosis is made, a multisystem investigation is required to investigate potential AVMs. It is also important to investigate family members for HHT due to its autosomal dominant inheritance pattern. Unexplained presentation, such as a prolonged asthma exacerbation in this case presentation, with significant family history should make one suspicious of the diagnosis. Since AVMs can be present in multiple organ systems, a multidisciplinary approach involving specialists in hematology, pulmonology, gastroenterology, interventional radiology, cardiology, neurology, and genetics may be required to provide optimal care for affected patients.

\section{REFERENCES}

${ }^{1}$ McDonald J, Bayrak-Toydemir P, Pyeritz RE. Hereditary hemorrhagic telangiectasia: An overview of diagnosis, management, and pathogenesis. Genet Med 2011; 13(7):607-616. PMID: 21546842.

${ }^{2}$ Kim YH, Vu PN, Choe SW, et al. Overexpression of activin receptor-like kinase 1 in endothelial cells suppresses development of arteriovenous malformations in mouse models of hereditary hemorrhagic telangiectasia. Circ Res 2020; 127(9):1122-1137. PMID: 32762495.

${ }^{3}$ Kühnel T, Wirsching K, Wohlgemuth W, Chavan A, Evert K, Vielsmeier V. Hereditary hemorrhagic telangiectasia. Otolaryngol Clin North Am 2018; 5l(1):237-254. PMID: 29217066.

4 Donaldson JW, McKeever TM, Hall IP, Hubbard RB, Fogarty AW. The UK prevalence of hereditary haemorrhagic telangiectasia and its association with sex, socioeconomic status and region of residence: A population-based study. Thorax 2014; 69(2):161-167. PMID: 24188926.

${ }_{5}^{5}$ Faughnan ME, Palda VA, Garcia-Tsao G, et al. International guidelines for the diagnosis and management of hereditary haemorrhagic telangiectasia. $\mathrm{J}$ Med Genet 2011; 48(2):73-87. PMID: 19553198.

${ }^{6}$ Lacombe P, Lacout A, Marcy PY, et al. Diagnosis and treatment of pulmonary arteriovenous malformations in hereditary hemorrhagic telangiectasia: An overview. Diagn Interv Imaging 2013; 94(9):835-848. PMID 23763987.

7 Guttmacher AE, Marchuk DA, White RI Jr. Hereditary hemorrhagic telangiectasia. N Engl J Med 1995; 333(14):918-924. PMID: 7666879.

8 Plauchu H, de Chadarévian JP, Bideau A, Robert JM. Age-related clinical profile of hereditary hemorrhagic telangiectasia in an epidemiologically recruited population. Am J Med Genet 1989; 32(3):291-297. PMID: 2729347.

9 Khurshid I, Downie GH. Pulmonary arteriovenous malformation. Postgrad Med J 2002; 78(918):191-197. PMID: 11930021.

${ }^{10}$ Dines DE, Arms RA, Bernatz PE, Gomes MR. Pulmonary arteriovenous fistulas. Mayo Clin Proc 1974; 49(7):460-465. PMID: 4834927.

${ }^{11}$ Labeyrie PE, Courthéoux P, Babin E, Bergot E, Touzé E, Pelage JP. Neurological involvement in hereditary hemorrhagic telangiectasia. J Neuroradiol 2016; 43(4):236-245. PMID: 27059009.

${ }^{12}$ van Gent MWF, Velthuis S, Post MC, et al. Hereditary hemorrhagic telangiectasia: How accurate are the clinical criteria? Am J Med Genet A2013; 161A(3):461-466. PMID: 23401183.

${ }^{13}$ Bayrak-Toydemir P, Mao R, Lewin S, McDonald J. Hereditary hemorrhagic telangiectasia: An overview of diagnosis and management in the molecular era for clinicians. Genet Med 2004; 6(4):175-191. PMID: 15266205.

${ }^{14}$ Ianora AAS, Memeo M, Sabba C, Cirulli A, Rotondo A, Angelelli G. Hereditary hemorrhagic telangiectasia: Multi-detector row helical CT assessment of hepatic involvement. Radiology 2004;230(1):250-259. PMID: 14645886.
KANSAS JOURNAL of MEDICINE HEREDITARY HEMORRHAGICTELANGIECTASIA continued.

${ }^{15}$ Bernard G, Mion F, Henry L, Plauchu H, Paliard P. Hepatic involvement in hereditary hemorrhagic telangiectasia: Clinical, radiological, and hemodynamic studies of 11 cases. Gastroenterology 1993; 105(2):482-487. PMID: 8335203.

${ }^{16}$ Shovlin CL, Guttmacher AE, Buscarini E, et al. Diagnostic criteria for hereditary hemorrhagic telangiectasia (Rendu-Osler-Weber syndrome). Am J Med Genet 2000; 91(1):66-67. PMID: 10751092.

${ }^{17}$ McDonald J, Bayrak-Toydemir P, DeMille D, Wooderchak-Donahue W, Whitehead K. Curaçao diagnostic criteria for hereditary hemorrhagic telangiectasia is highly predictive of a pathogenic variant in ENG or ACVRL1 (HHTl and HHT2). Genet Med 2020; 22(7):1201-1205. PMID: 32300199.

Keywords: hereditary hemorrhagic telangiectasia, asthma exacerbation, arteriovenous malformations 